\title{
Alcohol, stem cells and cancer
}

\author{
Shoujun Gu ${ }^{1}$, Bao-Ngoc Nguyen ${ }^{1}$, Shuyun Rao ${ }^{1}$, Shulin Li $^{2}$, Kirti Shetty ${ }^{3}$, Asif \\ Rashid ${ }^{4}$, Vivek Shukla ${ }^{5}$, Chu-Xia Deng ${ }^{1,6}$, Lopa Mishraa ${ }^{1,7}$ and Bibhuti Mishra ${ }^{1}$ \\ ${ }^{1}$ Department of Surgery, Center for Translational Medicine, George Washington University, Washington, DC, USA \\ 2 Departments of Pediatrics, The University of Texas MD Anderson Cancer Center, Houston, Texas, USA \\ ${ }^{3}$ Division of Gastroenterology and Hepatology, Johns Hopkins University School of Medicine, Baltimore, Maryland, USA \\ ${ }^{4}$ Departments of Gastroenterology and Liver Pathology, The University of Texas MD Anderson Cancer Center, Houston, \\ Texas, USA \\ ${ }^{5}$ Thoracic and Gastrointestinal Oncology Branch, National Cancer Institute, Bethesda, Maryland, USA \\ ${ }^{6}$ Faculty of Health Sciences, University of Macau, Macau SAR, China \\ ${ }^{7}$ Surgical Service, Veterans Affairs Medical Center, Washington DC, USA \\ Correspondence to: Bibhuti Mishra, email: neuralstem@gmail.com \\ Lopa Mishra, email: Imishra@gwu.edu \\ Chu-Xia Deng, email: cxdeng@umac.mo
}

Keywords: alcohol; cancer; stem cells; TGF- $\beta$; DNA repair

Received: September 19, $2017 \quad$ Accepted: October 17, 2017

Published: November 11, 2017

Copyright: Gu et al. This is an open-access article distributed under the terms of the Creative Commons Attribution License (CC-BY), which permits unrestricted use, distribution, and reproduction in any medium, provided the original author and source are credited.

ABSTRACT

Dosage, gender, and genetic susceptibility to the effects of alcohol remained only partially elucidated. In this review, we summarize the current knowledge of the mechanisms underlying the role of alcohol in liver and gastrointestinal cancers. In addition, two recent pathways- DNA repair and TGF- $\beta$ signaling which provide new insights into alcohol in the regulation of cancers and stem cells are also discussed here.

\section{INTRODUCTION}

Normal processing of endogenous as well as environmental factors and agents such as alcohol, is central to cellular homeostasis, and the deregulation of these pathways leads to liver and gastrointestinal inflammation and injury- often caused by a complex interplay between genetic and environmental factors [1]. In addition, protection from agents such as aldehydes generated spontaneously during cell metabolism is vital to normal cell function and tumor suppression $[2,3]$. Until recently, dosage, gender and genetic susceptibility to the effects of alcohol remained only partially elucidated. For instance, women are known to be more susceptible than men, yet the specific populations and the underlying genetic mechanisms of alcohol susceptibility remain unclear $[4,5]$. Multiple studies have examined the role of alcohol as a causative agent in multiple cancer types, that include breast, colon, esophageal, prostate and others [1, 6-9]. Yet few have been conducted prospectively, and the role of alcohol and its dosage in liver and gastrointestinal cancer is not completely understood $[10,11]$.

Although alcohol and illicit drugs are considered as different contributors to the global burden of disease, combined use of these substances is not an uncommon practice. Studies showed positive correlations between the incidence of alcohol and drug use [12-15]. A recent IARC (International Agency for Research on Cancer) meta-analysis examining the risk of esophageal cancer revealed the highest risk to be among those with concurrent alcohol and tobacco users, whereas the risk of esophageal cancer in alcohol users in the absence of tobacco usage was relatively lower [16]. Nevertheless, the risk of cancer is proportional to the dosage of used alcohol as many studies showed that compared to nondrinkers and occasional drinkers, the pooled relative risk (RR) was 1.03 for any, 0.97 for light, 1.04 for moderate, and 1.21 for heavy drinkers. As a matter of fact, the RR for heavy drinkers compared to nondrinkers and occasional drinkers was 5.13 for oral and pharyngeal cancer, 4.95 for oesophageal squamous cell carcinoma, 1.44 for colorectal, 2.65 for laryngeal and 1.61 for breast cancer. Heavy 
drinkers also had a significantly higher risk of cancer of the stomach, gallbladder, pancreas and lung [17]. In liver cancer, compared to nondrinker, the pooled RR was 0.91 for moderate drinking and 1.16 for heavy drinking [18]. Furthermore, for heavy drinkers, the pooled estimate RR was apparently higher for men than for women [19].

Alcohol is metabolized by alcohol dehydrogenases (ADHs) and aldehyde dehydrogenases (ALDHs). A review of case controlled studies examining genetic polymorphisms of the genes encoding these enzymes reveal a significant association between esophageal cancer risk and low $A D H 1 B$ and $A L D H 2$ genotypes, especially in east Asian heavy drinkers [20, 21]. However, specific populations of high-risk individuals have yet to be clearly defined. Two recent pathways- one in DNA repair pathway and the other in TGF- $\beta$ signaling have provided new insights that we discuss more broadly here [22, 23$]$.

\section{ALCOHOL AND DNA REPAIR PATHWAYS}

Since the discovery of DNA structure over 50 years ago, over 700 proteins and 900 distinct phosphorylation events have been described in the DNA damage response, reflecting the massive investment, cells make to preserve genomic integrity $[24,25]$. When one considers, that ultraviolet light from sunlight induces up to 105 DNA lesions per cell per day, then the need for sensors is enormous. The repair mechanism involves at least six pathways that cover the specific steps involved in multiple DNA lesions [26, 27]. One of the most formidable of post replication DNA lesions, is the replication fork lesion, a barrier to chromosome duplication, which leads to mitotic catastrophe, complex chromosome rearrangements, and cell death. These lesions are managed by inter-strand cross link (ICL) repair systems to prevent replication fork progression [28]. The central components of the incisional and trans-lesional synthesis steps of the ICL system are the Fanconi complex, an E3 ligase, and at least four other factors. Fanconi anemia is a cancer pre-disposition syndrome characterized by hypersensitivity to DNA interstrand cross-linking agents [29].

The thirteen Fanconi anemia(FA) complementation members act in a common pathway that result in DNA repair by homologous recombination. Replicationdependent ICL repair involves nucleolytic incisions flanking the ICL on one strand, trans-lesional DNA synthesis across the unhooked ICL, removal of the ICL by additional incisions, and homologous recombination. The central complex in this pathway is formed by the Fanconi anemia complementation group D2 (Fancd2), a core component of the Fanconi anemia complex, and

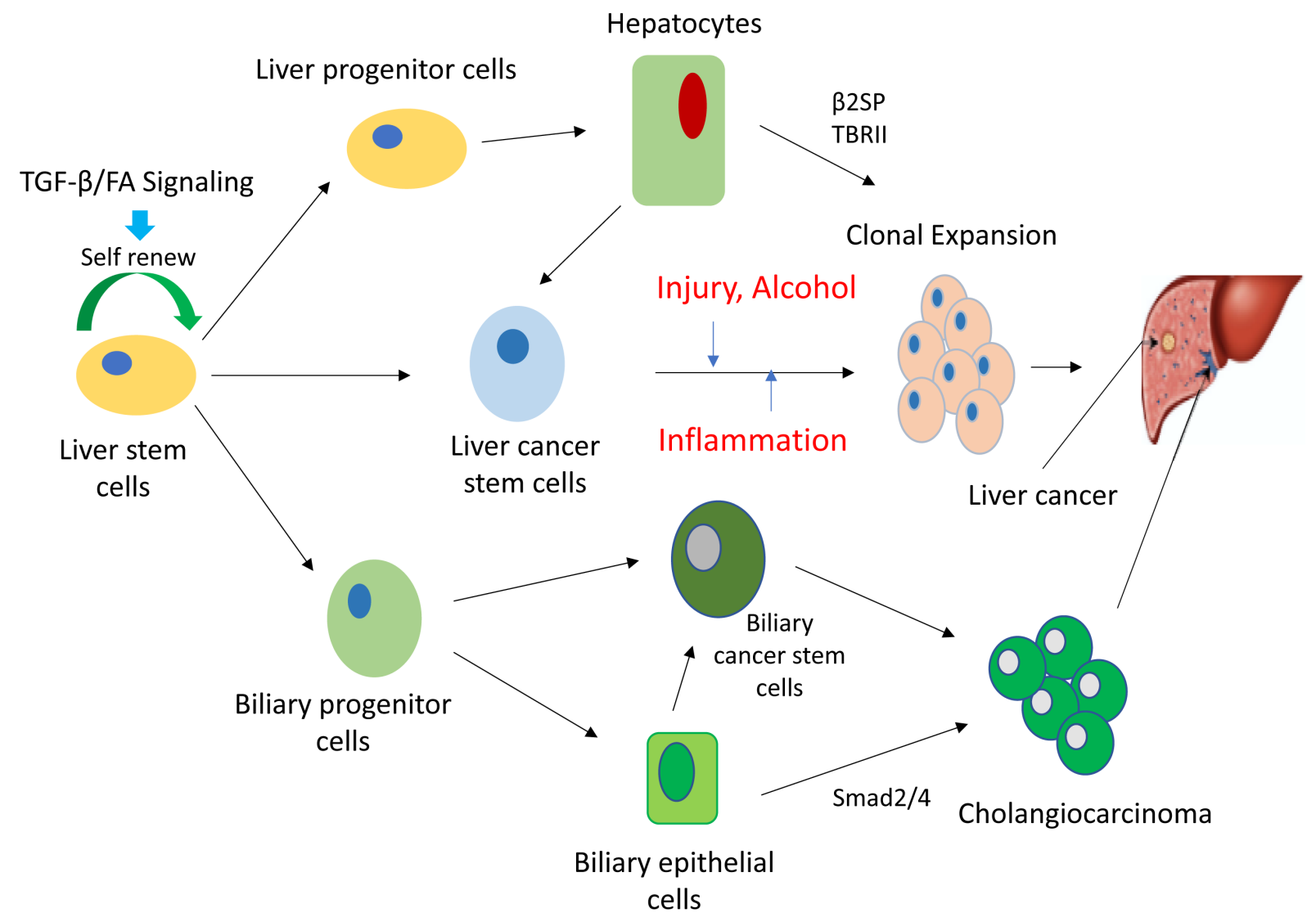

Figure 1: Schematic diagram of TGF- $\beta$ /FA pathway dependent regulation on liver stem cells. 
FAI (FANCI) proteins forming the FANC1-FANCD2 (ID) complex that are phosphorylated by ATR (ataxia telangiectasia and Rad3-related). FA is caused by biallelic mutations of fifteen members of FANC pathway with inability to respond to cellular stress and ensuing DNA damage during $\mathrm{S}$ phase and loss of genome integrity $[30,31]$. Patients with FA frequently develop bone marrow failure requiring allogeneic hematopoietic stem cell (HSC) transplantation, and have developmental abnormalities (short stature, a triangular face, and thumb abnormalities) $[31,32]$. They also have a high risk of developing myelodysplasia (MDS), acute myeloid leukemia (AML) $[31,33-36]$, and hepatocellular carcinoma (HCC), especially with androgen treatment [37]. However, FANCD2 knockout mice do not develop HCC. Therefore, while the Fanconi anemia pathway is implicated in maintaining hematopoietic stem cell homeostasis, its role in liver stem cells and cancer remains unclear.

The development of cancer due to the failure of response to agents such as alcohol producing reactive aldehydes, creating adducts that directly bind and damage DNA, has recently been observed in models with genetic inactivation of the Fanconi anemia members [23]. Fanc mutant intercrosses with $A L D H 2$ mutant mice are susceptible to ethanol teratogenicity and defective DNA inter-strand cross link repair [23, 38]. Yet, mice with Fanc mutants (on their own) treated with alcohol do not develop any fetal-alcohol like aberrations suggesting a more complex process is involved in toxin induced DNA damage [2, 39-41] and that the essential sensors and the mechanisms for aberrant DNA damage from alcohol remain unclear.

\section{ALCOHOL AND TGF-B PATHWAY}

TGF- $\beta$ and the Fanconi anemia pathway, two critical pathways involved in both stem cell maintenance as well as differentiation, have also been shown to play a pivotal role in metabolizing alcohol. TGF- $\beta$ serves as an essential regulator of cell polarity, growth, differentiation, and lineage specificity as well as a tumor suppressor pathway in multiple cell types $[42,43]$. Defective TGF- $\beta$ signaling is implicated in liver injury, inflammation and multiple cancers owing to the frequent somatic mutations in, or deregulation of, its components, such as Smad3, Smad4, and TGF- $\beta$ receptors 1 and 2 (TBR1 and TBR2) (Figure 1). Smads are the intracellular mediators of TGF- $\beta$ signaling [44-47], and their function is modulated by adaptor proteins such as the Smad anchor for receptor activation, filamin, microtubules, and $\beta 2$-spectrin ( $\beta 2 \mathrm{SP}$, gene Sptbn1) [48-50].

TGF- $\beta$-activated Smads also orchestrate specific histone modifications and chromatin remodeling to activate their transcriptional targets. Keratinocytes cultured from TGF $\beta 1$-null mice have marked genomic instability that could accelerate tumor progression [51]. More recently, studies in the Smad4 conditional knockout mice that develop head and neck cancers, demonstrate a key role for Smad4 as a guardian of the genome through regulation of the Fanconi anemia/Brca (Fanc/Brca) DNA repair pathway $[52,53]$.

The major role of $\beta 2 \mathrm{SP}$ in maintaining genomic stability following alcohol-induced DNA damage is supported by the fact that $\beta 2 \mathrm{SP}$ defective mouse embryos display some symptom of human fetal alcohol syndrome (Figure 2) [22]. Furthermore, the development

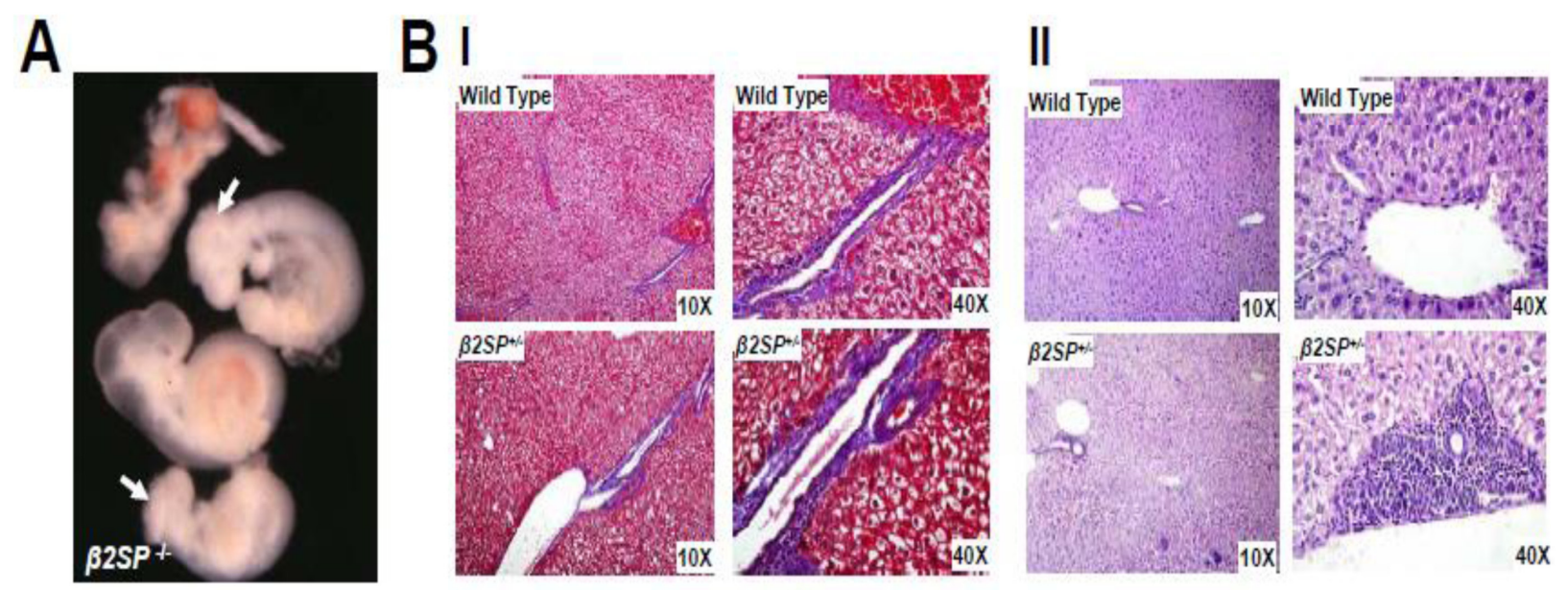

Figure 2: A. $\beta 2 \mathrm{SP}^{-/ /}$mouse embryos display some symptom of human fetal alcohol syndrome, microcephaly (white arrow), anencephaly and anophthalmia; B. Alcoholic hepatitis is induced by alcohol in $\beta 2 \mathrm{SP}^{+/-}$mice compared to wild type normal controls. Alcoholic hepatitislike picture in $\beta 2 \mathrm{SP}$ mutant mice: effect of alcohol on $\beta 2 \mathrm{SP}^{+/-}$mice liver. Mice were treated with alcohol at dose of 30ml/day. I) Masson's Trichrome stain for alcohol treatment group; II) H\&E stain for alcohol treatment group. 
of hepatocellular cancers (HCCs) in $\beta 2$ SP heterozygote mutants establishes $\beta 2 \mathrm{SP}$ as a functional tumor suppressor. As a matter of fact, $\beta 2$ SP have been observed to associate with Fanconi proteins (G and D) as well as with DNA interstrand cross-links [23, 41]. In addition, Smads have been identified as regulators of Fanconi genes [54]. Similarly, by virtue of its involvement in Smad3/4 localization and subsequent activation of $\mathrm{Smad} 3 / 4, \beta 2 \mathrm{SP}$ may enhance TGF- $\beta$ tumor suppressor function. Indeed, loss of $\beta 2 \mathrm{SP}$ leads to decreased Fancd 2 levels and sensitizes $\beta 2 \mathrm{SP}$ mutants to DNA damage by ethanol treatment, leading to phenotypes that closely resemble those observed in animals lacking both $A L D H 2$ and Fancd 2 and resemble human fetal alcohol syndrome. Sptbn1-deficient cells are hypersensitive to DNA crosslinking agents and have defective DNA double-strand break repair that is rescued by ectopic Fancd 2 expression. Taken together, TGF- $\beta$ / $\beta 2 \mathrm{SP}$ signaling acts as a potential guardian of genomic stability from genotoxic metabolites through modulation of the Fanconi anemia DNA repair pathway, yet the exact mechanisms remain to be elucidated.

\section{ALCOHOL AND TLR4}

TLR4 is a receptor for endotoxin which participates in many inflammatory processes such as M1 activation of hepatic macrophages in alcoholic liver disease [55]. However, its role in liver carcinogenesis via ectopic expression and activation has only recently been revealed in alcohol/HCV-associated HCC models [55]. Alcohol feeding to mice expressing the HCV Ns5a in a hepatocyte specific manner aggravates liver inflammation via activation of overexpressed TLR4 in the parenchymal cells [56]. Long-term alcohol feeding produces liver tumors in these transgenic mice in a manner dependent on TLR4. From these mice, tumor-initiating stem celllike cells (TICs) have been isolated. These TICs exhibit self-renewal and tumorigenic activities driven by TLR4dependent upregulation of the stem cell factor NANOG. A defective TGF- $\beta$ tumor suppressor pathway is identified in the TICs and mediated by NANOG target genes IGF2BP3 and YAP1. Conversely, mice with an attenuated TGF- $\beta$ pathway due to haploinsufficiency of $\beta 2$ - Spectrin, spontaneously develop liver tumors and alcohol feeding increases tumor incidence in a TLR4-dependent manner [57]. This reciprocal antagonism between TLR4 and TGF- $\beta$ pathways may serve as a novel therapeutic target for HCC.

Whilst the phenotypes in the TGF- $\beta$ deficient mutant mice are dramatic and restricted to specific compartments [58-60], it is clear that multiple tiers of control are present in the human disease- LOI at chromosome 11 leading to raised levels of TERT, IGF2, etc. The heterozygous TGF- $\beta$ deficient mutants develop cancers spontaneously on a C57BL/ 6 background and in this regard the heterozygotes resemble sporadic cancer formation in humans [61-63]. There are limitations to all models, but over the last few years many of these have been selected and have been continually refined to optimize readouts. This has been successful to date and enabled the identification of new fundamental elements of two-tier control [64-66]. Finally, there are well established models of tumorigenesis available to study cancer progression in mice, as well as xenografts of cancer cell lines into recipients that many scientists have been able to employ successfully [57, 67].

\section{CONCLUSION}

Collectively, the alcohol dosage and specific risk factors of alcohol use as the underlying cause of cancer remain unclear. Therefore, there is an urgent need for strong fundamental and clinical studies to examine the specific dosage, sex and genetic risk factors that confer cancer risk to alcohol intake.

\section{Abbreviations}

IARC: International Agency for Research on Cancer; RR: Relative Risk; ADH: Alcohol Dehydrogenases; ALDH: Aldehyde Dehydrogenase; ICL: Interstrand Cross Link; HSC: Hematopoietic Stem Cell; MDS: Myelodysplasia; AML: Acute Myeloid Leukemia; HCC: Hepatocellular Carcinoma; TGF- $\beta$ : Transforming Growth Factor beta; $\beta 2$ SP: $\beta 2$-spectrin; Fanc: Fanconi Anemia; LOI: Loss of Imprint; TLR4: Toll-like Receptor 4

\section{Author contributions}

The review was written by SG, BM and LM. Strong intellectual input was provided by $\mathrm{BN}, \mathrm{SR}, \mathrm{SL}, \mathrm{KS}, \mathrm{AR}$, VS and CD.

\section{ACKNOWLEDGMENTS}

We acknowledge Nilesh Dubey for helping in liver extraction and immunohistochemical staining.

\section{CONFLICT OF INTEREST}

None.

\section{FUNDING}

This work was supported by NIH grants R01AA023146 (L. Mishra), P01CA130821 (L. Mishra), VA Merit I01BX003732 (L. Mishra), R01CA120895 (S. Li) and R01DK102767-01A1 (S. Li). 


\section{REFERENCES}

1. Zakhari, S., Chronic alcohol drinking: Liver and pancreatic cancer? Clin Res Hepatol Gastroenterol, 2015. 39 Suppl 1: p. S86-91.

2. Seitz, H.K. and F. Stickel, Molecular mechanisms of alcohol-mediated carcinogenesis. Nat Rev Cancer, 2007. 7: p. 599-612.

3. Matejcic, M., M.J. Gunter, and P. Ferrari, Alcohol Metabolism and Oesophageal Cancer: A Systematic Review of the Evidence. Carcinogenesis, 2017.

4. Mishra, L., et al., More rapid elimination of alcohol in women as compared to their male siblings. Alcohol Clin Exp Res, 1989. 13: p. 752-4.

5. Sato, N., et al., Sex difference in alcohol-related organ injury. Alcohol Clin Exp Res, 2001. 25: p. 40S-45S.

6. Bagnardi, V., et al., Light alcohol drinking and cancer: a meta-analysis. Ann Oncol, 2013. 24: p. 301-8.

7. Zakhari, S. and J.B. Hoek, Alcohol and breast cancer: reconciling epidemiological and molecular data. Adv Exp Med Biol, 2015. 815: p. 7-39.

8. Ratna, A. and P. Mandrekar, Alcohol and Cancer: Mechanisms and Therapies. Biomolecules, 2017. 7.

9. Na, H.K. and J.Y. Lee, Molecular Basis of Alcohol-Related Gastric and Colon Cancer. Int J Mol Sci, 2017. 18.

10. Lakshman, R., et al., Is alcohol beneficial or harmful for cardioprotection? Genes Nutr, 2010. 5: p. 111-20.

11. Zakhari, S. and T.K. Li, Determinants of alcohol use and abuse: Impact of quantity and frequency patterns on liver disease. Hepatology, 2007. 46: p. 2032-9.

12. Degenhardt, L., et al., Toward a global view of alcohol, tobacco, cannabis, and cocaine use: findings from the WHO World Mental Health Surveys. PLoS Med, 2008. 5: p. e141.

13. Hall, W., et al., The 12-month prevalence of substance use and ICD-10 substance use disorders in Australian adults: findings from the National Survey of Mental Health and Well-Being. Addiction, 1999. 94: p. 1541-50.

14. Stinson, F.S., et al., Comorbidity between DSM-IV alcohol and specific drug use disorders in the United States: results from the National Epidemiologic Survey on Alcohol and Related Conditions. Drug Alcohol Depend, 2005. 80: p. 105-16.

15. Hedden, S.L., et al., Patterns of illegal drug use among an adult alcohol dependent population: results from the National Survey on Drug Use and Health. Drug Alcohol Depend, 2010. 106: p. 119-25.

16. Shingler, E., et al., Tobacco and alcohol cessation or reduction interventions in people with oral dysplasia and head and neck cancer: systematic review protocol. Syst Rev, 2017. 6: p. 161 .

17. Bagnardi, V., et al., Alcohol consumption and site-specific cancer risk: a comprehensive dose-response meta-analysis Br J Cancer, 2015. 112: p. 580-93.
18. Turati, F., et al., Alcohol and liver cancer: a systematic review and meta-analysis of prospective studies. Ann Oncol, 2014. 25: p. 1526-35.

19. Cai, S., et al., Alcohol drinking and the risk of colorectal cancer death: a meta-analysis. Eur J Cancer Prev, 2014. 23: p. 532-9.

20. Yang, S.J., et al., Relationship between genetic polymorphisms of ALDH2 and ADH1B and esophageal cancer risk: a meta-analysis. World J Gastroenterol, 2010. 16: p. 4210-20.

21. Huang, C.C., et al., Investigating the Association between Alcohol and Risk of Head and Neck Cancer in Taiwan. Sci Rep, 2017. 7: p. 9701.

22. Chen, J., et al., Loss of the transforming growth factorbeta effector beta2-Spectrin promotes genomic instability. Hepatology, 2017. 65: p. 678-693.

23. Langevin, F., et al., Fancd 2 counteracts the toxic effects of naturally produced aldehydes in mice. Nature, 2011. 475: p. $53-8$.

24. Ciccia, A. and S.J. Elledge, The DNA damage response: making it safe to play with knives. Mol Cell, 2010. 40: p. 179-204.

25. Jackson, S.P. and J. Bartek, The DNA-damage response in human biology and disease. Nature, 2009. 461: p. 1071-8.

26. Huertas, P., DNA resection in eukaryotes: deciding how to fix the break. Nat Struct Mol Biol, 2010. 17: p. 11-6.

27. Hoeijmakers, J.H., DNA damage, aging, and cancer. N Engl J Med, 2009. 361: p. 1475-85.

28. Deans, A.J. and S.C. West, DNA interstrand crosslink repair and cancer. Nat Rev Cancer, 2011. 11: p. 467-80.

29. Joenje, H. and K.J. Patel, The emerging genetic and molecular basis of Fanconi anaemia. Nat Rev Genet, 2001. 2: p. $446-57$

30. de Winter, J.P. and H. Joenje, The genetic and molecular basis of Fanconi anemia. Mutat Res, 2009. 668: p. 11-9.

31. Dokal, I., Fanconi anemia is a highly penetrant cancer susceptibility syndrome. Haematologica, 2008. 93: p. 4868.

32. Becker, P.S., et al., Preclinical correction of human Fanconi anemia complementation group A bone marrow cells using a safety-modified lentiviral vector. Gene Ther, 2010. 17: p. 1244-52.

33. Bagby, G.C. and G. Meyers, Bone marrow failure as a risk factor for clonal evolution: prospects for leukemia prevention. Hematology Am Soc Hematol Educ Program, 2007: p. 40-6.

34. Kutler, D.I., et al., High incidence of head and neck squamous cell carcinoma in patients with Fanconi anemia. Arch Otolaryngol Head Neck Surg, 2003. 129: p. 106-12.

35. Quentin, S., et al., Myelodysplasia and leukemia of Fanconi anemia are associated with a specific pattern of genomic abnormalities that includes cryptic RUNX1/AML1 lesions. Blood, 2011. 117: p. e161-70. 
36. Soulier, J., Fanconi anemia. Hematology Am Soc Hematol Educ Program, 2011. 2011: p. 492-7.

37. Masserot-Lureau, C., et al., Incidence of liver abnormalities in Fanconi anemia patients. Am J Hematol, 2012. 87: p. 547-9.

38. Van Wassenhove, L.D., D. Mochly-Rosen, and K.I. Weinberg, Aldehyde dehydrogenase 2 in aplastic anemia, Fanconi anemia and hematopoietic stem cells. Mol Genet Metab, 2016. 119: p. 28-36.

39. Moldovan, G.L. and A.D. D'Andrea, To the rescue: the Fanconi anemia genome stability pathway salvages replication forks. Cancer Cell, 2012. 22: p. 5-6.

40. Joo, W., et al., Structure of the FANCI-FANCD2 complex: insights into the Fanconi anemia DNA repair pathway. Science, 2011. 333: p. 312-6.

41. Oberbeck, N., et al., Maternal aldehyde elimination during pregnancy preserves the fetal genome. Mol Cell, 2014. 55: p. 807-17.

42. Massague, J., S.W. Blain, and R.S. Lo, TGFbeta signaling in growth control, cancer, and heritable disorders. Cell, 2000. 103: p. 295-309.

43. Mullen, A.C., et al., Master transcription factors determine cell-type-specific responses to TGF-beta signaling. Cell, 2011. 147: p. 565-76.

44. Moses, H.L. and R. Serra, Regulation of differentiation by TGF-beta. Curr Opin Genet Dev, 1996. 6: p. 581-6.

45. Shi, Y. and J. Massague, Mechanisms of TGF-beta signaling from cell membrane to the nucleus. Cell, 2003. 113: p. 685700 .

46. Zawel, L., et al., Human Smad3 and Smad4 are sequencespecific transcription activators. Mol Cell, 1998. 1: p. 611-7.

47. Shi, Y., et al., Crystal structure of a Smad MH1 domain bound to DNA: insights on DNA binding in TGF-beta signaling. Cell, 1998. 94: p. 585-94.

48. Tang, Y., et al., Disruption of transforming growth factorbeta signaling in ELF beta-spectrin-deficient mice. Science, 2003. 299: p. 574-7.

49. Wu, G., et al., Structural basis of Smad2 recognition by the Smad anchor for receptor activation. Science, 2000. 287: p. 92-7.

50. Mishra, L. and B. Marshall, Adaptor proteins and ubiquinators in TGF-beta signaling. Cytokine Growth Factor Rev, 2006. 17: p. 75-87.

51. Glick, A., et al., Defects in transforming growth factorbeta signaling cooperate with a Ras oncogene to cause rapid aneuploidy and malignant transformation of mouse keratinocytes. Proc Natl Acad Sci U S A, 1999. 96: p. 14949-54.

52. Bornstein, S., et al., Smad4 loss in mice causes spontaneous head and neck cancer with increased genomic instability and inflammation. J Clin Invest, 2009. 119: p. 3408-19.

53. Korc, M., Smad4: gatekeeper gene in head and neck squamous cell carcinoma. J Clin Invest, 2009. 119: p. 320811.

54. Meier, D. and D. Schindler, Fanconi anemia core complex gene promoters harbor conserved transcription regulatory elements. PLoS One, 2011. 6: p. e22911.

55. Tsukamoto, H., L. Mishra, and K. Machida, Alcohol, TLR4TGF-beta antagonism, and liver cancer. Hepatol Int, 2014. 8 Suppl 2: p. 408-12.

56. Machida, K., et al., Toll-like receptor 4 mediates synergism between alcohol and HCV in hepatic oncogenesis involving stem cell marker Nanog. Proc Natl Acad Sci U S A, 2009. 106: p. $1548-53$

57. Chen, C.L., et al., Reciprocal regulation by TLR4 and TGFbeta in tumor-initiating stem-like cells. J Clin Invest, 2013. 123: p. 2832-49.

58. Chen, J., et al., TGF-beta/beta2-spectrin/CTCF-regulated tumor suppression in human stem cell disorder BeckwithWiedemann syndrome. J Clin Invest, 2016. 126: p. 527-42.

59. Chen, J., et al., Vitamin D Deficiency Promotes Liver Tumor Growth in Transforming Growth Factor-beta/ Smad3-Deficient Mice Through Wnt and Toll-like Receptor 7 Pathway Modulation. Sci Rep, 2016. 6: p. 30217.

60. Tang, Y., et al., Progenitor/stem cells give rise to liver cancer due to aberrant TGF-beta and IL-6 signaling. Proc Natl Acad Sci U S A, 2008. 105: p. 2445-50.

61. Majumdar, A., et al., Hepatic stem cells and transforming growth factor beta in hepatocellular carcinoma. Nat Rev Gastroenterol Hepatol, 2012. 9: p. 530-8.

62. Tang, Y., et al., Transforming growth factor-beta suppresses nonmetastatic colon cancer through Smad4 and adaptor protein ELF at an early stage of tumorigenesis. Cancer Res, 2005. 65: p. 4228-37.

63. Katuri, V., et al., Inactivation of ELF/TGF-beta signaling in human gastrointestinal cancer. Oncogene, 2005. 24: p. 8012-24.

64. Chen, J., et al., Mutational Profiles Reveal an Aberrant TGF-beta-CEA Regulated Pathway in Colon Adenomas. PLoS One, 2016. 11: p. e0153933.

65. Yao, Z.X., et al., Epigenetic silencing of beta-spectrin, a TGF-beta signaling/scaffolding protein in a human cancer stem cell disorder: Beckwith-Wiedemann syndrome. J Biol Chem, 2010. 285: p. 36112-20.

66. Wang, R.H., et al., A role of SMAD4 in iron metabolism through the positive regulation of hepcidin expression. Cell Metab, 2005. 2: p. 399-409.

67. Lin, L., et al., The STAT3 inhibitor NSC 74859 is effective in hepatocellular cancers with disrupted TGF-beta signaling. Oncogene, 2009. 28: p. 961-72. 